\author{
Helena Bažec* \\ Università del Litorale
}

Georgia Milioni ${ }^{* *}$

Università Nazionale e Kapodistriaca di Atene

\title{
BIANCO, NERO E ROSSO NELLE ESPRESSIONI IDIOMATICHE IN ITALIANO, GRECO E SLOVENO: CONVERGENZE E DIVERGENZE
}

\begin{abstract}
Benché l'italiano, lo sloveno e il greco appartengano a famiglie linguistiche diverse, hanno tutti lo stesso antenato comune, l'indoeuropeo. Nel contributo si vogliono presentare in chiave contrastiva le origini etimologiche, la simbologia e l'influsso culturale che si manifestano nelle espressioni idiomatiche contenenti cromonimi con lo scopo specifico di indagare quale di questi criteri può avere avuto più influenza sul significato e quali lingue presentano maggiori somiglianze. Questa ricerca prende in considerazione i tre cromonimi più frequenti in base ai corpora linguistici; il nero, il bianco e il rosso e le relative traduzioni in tutte e tre le lingue indagate. L'analisi conduce alla conclusione che la contiguità territoriale, culturale e religiosa non ha avuto molta importanza, come pure non l'ha avuta l'etimologia. Le tre lingue condividono la simbologia di base, ma nella maggioranza dei casi presentano divergenze nell'uso. Lo sloveno e il greco, le lingue più lontane territorialmente e culturalmente, che non hanno contatti diretti, riscontrano il maggior numero di somiglianze. Segue la coppia di lingue italiano e greco e infine lo sloveno e l'italiano che hanno il minor numero di somiglianze.

Parole chiave: fraseologia contrastiva, cromonimi, espressioni idiomatiche, sloveno, greco, italiano.
\end{abstract}

\section{INTRODUZIONE}

Le lingue, come tutti gli organismi viventi, si adattano continuamente alle nuove esigenze della società. Tra tutti i livelli di analisi linguistica proprio il lessico e, di conseguenza le espressioni idiomatiche, riflettono

\footnotetext{
*helena.bazec@fhs.upr.si

**.gmilioni@ill.uoa.gr
} 
in misura maggiore le vicende storiche e gli influssi a cui una comunità di parlanti è esposta. Nuove espressioni affiorano quasi quotidianamente, mentre altre cadono in disuso fino a scomparire del tutto dalla lingua.

Nel presente contributo intendiamo analizzare le espressioni idiomatiche contenenti cromonimi in tre lingue della famiglia indoeuropea, appartenenti a gruppi diversi: l'italiano, una lingua romanza, lo sloveno, una lingua slava meridionale e il greco moderno, una lingua "isolata". Molti studi interlinguistici si sono basati sui cromonimi (cfr. Kocjančič 2019; Zeronikova, 2009), ma nessuno si è occupato delle espressioni idiomatiche nelle tre lingue in chiave contrastiva.

Diacronicamente parlando, il Greco e il latino, gli antenati del greco moderno (nel proseguo greco) e dell'italiano, vantano una vetustà ininterrotta fin dall'antichità. Queste due lingue sono state la culla della cultura occidentale e hanno ancora oggi «la funzione di potenti "serbatoi" per la "modellizzazione" di strutture linguistiche e per la diffusione di elementi lessicali e fraseologici comuni» (Banfi e Grandi 2003: 41). Già nell'età imperiale il Greco e il latino raggiunsero un alto livello di convergenza linguistica e culturale condividendo oltre che comuni modelli culturali pure il messaggio cristiano (Banfi e Grandi 2003). Ma nell'Alto Medio Evo questa unità si spezzò - anche a causa della migrazione massiccia di popolazioni slave nei territori di confine - dando origine a due realtà: quella latinoromana e quella greco-bizantina. In questo quadro, lo slavo meridionale rappresenta un ponte sia culturale che linguistico tra Oriente e Occidente, tra la tradizione greca e quella latina. Quindi «già a partire dal X secolo, l'Europa medievale ebbe tre grandi lingue veicolari: il latino, il greco e il paleo-slavo. Ognuna di esse, strumento di culture nate da una matrice comune e successivamente distanziatesi» (Banfi e Grandi 2003: 45).

Collocando queste lingue nel quadro linguistico e politico dell'Europa contemporanea, vediamo che l'italiano è a stretto contatto con lo sloveno sul confine orientale, mentre non ha più legami territoriali diretti con la Grecia. Lo sloveno è una lingua slava che si colloca nell'estrema periferia ovest del diasistema slavo ed è fin dal periodo della migrazione delle genti nell'orbita latino-romana, con la quale condivide la religione. È la lingua slava meridionale più distante dal greco moderno sia geograficamente che linguisticamente, in quanto non rientra nella lega linguistica balcanica (Nocentini 2004), non usa l'alfabeto cirillico e non ha una presenza storicamente rilevante di religione ortodossa che avrebbe potuto influire significativamente sulla cultura e, di conseguenza, sulla lingua.

Da tali premesse avanziamo l'ipotesi di ricerca che anche nelle espressioni idiomatiche si rifletta ancora oggi il patrimonio storico comune presentando molte più convergenze che divergenze. Per verificare questa 
ipotesi cercheremo di scoprire se i cromonimi analizzati nelle tre lingue hanno un'etimologia comune, se la simbologia influisce sull'uso effettivo nella fraseologia e se la contiguità territoriale, culturale e religiosa significa necessariamente più convergenze. Cercheremo di trovare le risposte a questi quesiti in base a un'analisi contrastiva delle espressioni idiomatiche contenenti cromonimi. Dato che la presente ricerca è a uno stadio iniziale e per ragioni di spazio abbiamo deciso di concentrarci solo sui tre colori più frequenti nell'uso linguistico: il bianco, il nero e il rosso.

\section{I CROMONIMI}

Tutti gli esseri umani percepiscono i colori in modo uguale attraverso gli occhi, ma sono la nostra cultura e lingua che influiscono fortemente su questa percezione per cui il lessico di alcune lingue conosce solamente due cromonimi, mentre altre lingue ne distinguono dieci e più (Kay e Maffi 2013). Nel 1969 Berlin e Kay hanno provato che «la categorizzazione del colore non è un prodotto arbitrario della lingua, ma è guidata da fattori extralinguistici universali» (Basile et al 2010: 346). I due autori hanno individuato delle tendenze universali applicabili a tutte le lingue storico-naturali che risultano in un elenco di undici cromonimi di base i quali nascono secondo una gerarchia precisa (Schema 1). Questa teoria fu criticata aspramente, additata come "anglocentrica" (Philip 2003) e ampliata con l'aggiunta del dodicesimo colore di base perché in alcune lingue indoeuropee esistono due espressioni distinte per il blu, come ad esempio nell'italiano che accanto ad esso usa anche 'azzurro'.

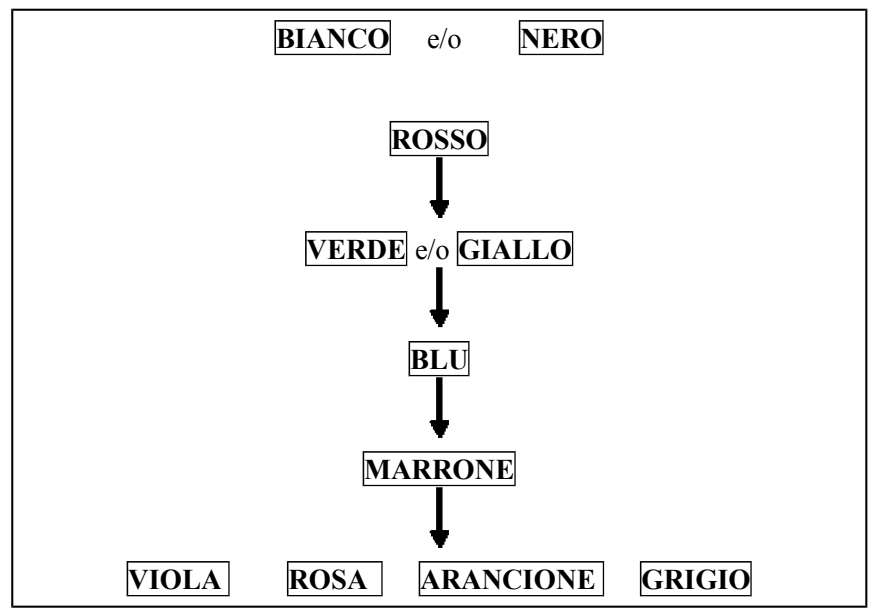

Schema 1. Ricostruzione delle categorie di colori secondo Berlin e Kay (1969: 4) 
Ciò nonostante, la teoria di Berlin e Kay rimane tutt'oggi un punto di riferimento per tutti i lavori scientifici legati ai colori. Le critiche poste alla teoria non hanno mai invalidato i suoi risultati, per cui partiremo da questa lista che compareremo con la frequenza d'uso. I dati estrapolati dai maggiori corpora ${ }^{1}$ provano che, da un punto di vista prettamente statistico, in sloveno e greco si colloca al primo posto il bianco, al secondo il nero e al terzo il rosso. In italiano, invece, il primo posto è occupato dal nero, seguito dal bianco e rosso. Possiamo supporre che questi tre cromonimi più usati avranno prodotto anche il maggior numero di espressioni idiomatiche, dato che una maggiore frequenza d'uso dovrebbe significare anche contesti d'uso più diversificati, una più ampia ricchezza semantica e, di conseguenza, maggiore potenziale fraseologico.

\subsection{Etimologia}

L'espressione per il colore bianco in italiano proviene dal germanico antico blanch che inizialmente significava 'scintillante come le armi di metallo' e in seguito ha cominciato ad indicare il colore (Daniele 2015). Lo sloveno bel deriva dalla radice ide. * $b^{h} e l(H)$ 'lucente, bianco' e ha lo stesso significato e uso come nello slavo ecclesiastico antico, e probabilmente anche nel protoslavo *bĕls (Snoj 2016). In greco il termine per bianco è $\alpha ́ \sigma \pi \rho o \varsigma$ e deriva dal greco medievale $\alpha ́ \sigma \pi \rho o(v)$ da $\alpha ́ \sigma \pi \rho o$ vó $\mu \iota \sigma \mu \alpha$, che indicava una moneta bianca, ovvero una moneta di piccolo valore. Così veniva denominata la moneta tagliata da poco perché la sua superficie era ancora ruvida. Similmente in latino asperumnummus indica una moneta di conio recente, nuova di zecca. Asperum, neutro di asper, deriva da una forma protoindoeuropea $* h_{2} e s p$ 'tagliare'.

Nero in italiano deriva dal lat. nigrum 'nero, scuro, oscuro, tragico' che a sua volta deriverebbe dalla radice gr. necros 'morto, luttuoso' (Daniele 2015). Gli etimologi non sono certi sull'etimologia della parola, ma potrebbe avere origini anche nell'ide. *nekw-t- 'notte' (Mattevi 2013). In sloveno črn deriva dallo slavo ecclesiastico antico (e protoslavo) čъrnъ-<ide. *kers- che indicava colori scuri, con l'aggiunta del suffisso *-no $\left({ }^{*} k_{0} s n o ́-\right)$ avrebbe il significato del colore (Snoj 2016). Nella koinè nero è detto $\mu \alpha \tilde{v} \rho o \varsigma / \mu \alpha v \rho o ́ \varsigma$

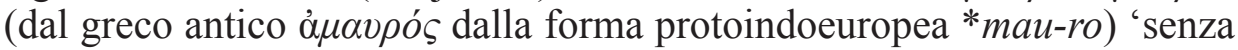
sole, nero, scuro'.

${ }^{1}$ Per lo sloveno è stato consultato il corpus Gigafida (304.357 occorrenze di bel 'bianco', 281.209 per črn 'nero' e 245.050 per rdeč 'rosso'), per l'italiano i dati si basano sul corpus della Repubblica (nero 74.474; bianco 54.445; rosso 46.424), sul CoLFIS e CORIS che confermano i dati; per il greco i dati sono stati ottenuti da $M \pi \alpha \mu \pi \imath v \iota \omega ́ \tau \eta \varsigma \Gamma(2011)$. 
L'aggettivo rosso trae le proprie origini etimologiche dal lat. russus che a sua volta deriva dalla radice ide. *reudh (Daniele 2015). L'aggettivo slo. rdeč deriva dallo slavo ecclesiastico antico, dove lo troviamo nella forma $r$ r děję, participio presente del verbo rzdêti 'diventare rosso, arrossire'. Anche questo riconduce alla stessa radice indoeuropea (Snoj 2016).

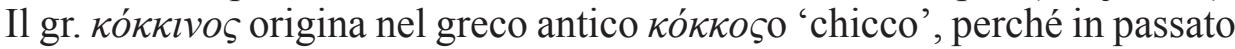
il colore rosso si produceva dai chicchi di quercia. L'aggettivo $\varepsilon \rho v \theta \rho o ́ \varsigma$ riconduce alla stessa radice indoeuropea * reudh.

\subsection{Simbologia}

Sin dall'antichità ogni popolo ha elaborato simbolismi cromatici diversi per interpretare e ordinare in qualche modo la molteplicità delle tonalità che ci circondano. Nella cultura occidentale il bianco è associato a valori positivi e in quanto tale spesso appare accanto al suo opposto, il nero (magia bianca/nera, angeli bianchi e demoni neri) (Kovačev 1997). Il bianco implica l'inizio, la nascita e l'origine (Musek 1990), spesso è simbolo dell'assoluto e dell'eternità, della perfezione e della bontà. Si associa alla delicatezza, grazia e semplicità, per cui in italiano viene usato anche come nome di donna. Si collega anche al freddo, perché è il colore inerente alla neve (Kovačev 1997). In qualità di portatore di connotazioni positive, il bianco è simbolo di religione, per questo motivo spesso a raffigurare metaforicamente il divino si trovano animali bianchi (colomba, agnello, unicorno) come portatori del messaggio di dio. Nel cristianesimo simboleggia anche la risurrezione, per questo motivo Gesù spesso è raffigurato in vesti bianche (Kovačev 1997). Come simbolo di immacolatezza si usa per vari riti religiosi (il battesimo, il matrimonio).

Il nero preserva il valore associatogli nell'antichità ed è quindi il colore della notte e dell'oscurità, per cui richiama il male e in generale i campi semantici legati al disagio (incidenti, morti, pessimismo, sciagura, illegalità e giudizio morale negativo), ma ha anche un lato neutrale, quando viene usato in riferimento a correnti politiche conservatrici, mentre nell'ultimo secolo assume un valore positivo quando diventa il colore dell'eleganza (autorità, distanza, inaccessibilità). Data la prevalente associazione negativa nelle lingue, questo cromonimo accostato ad altre parole evoca cose negative, cattive, sporche, spiacevoli, scure e illegali (Zeroníková 2009).

Stando a Kovačev (1997), il colore rosso è da sempre molto apprezzato. Sin dalle prime civiltà umane si usava per i vestiti dei più alti funzionari dello stato e della religione. Nel passato più recente era simbolo del movimento dei lavoratori, del comunismo e del socialismo. Il rosso, però, è anche il colore del sangue e del fuoco e in parte è sinonimo anche di alcuni sentimenti forti, come l'amore, l'odio, l'imbarazzo e l'ira. Oltre a questo, indica un pericolo 
o una proibizione. È anche il colore della guerra e del dio della guerra Marte, dell'aggressività per il suo legame con la lotta, ma anche della solidarietà e dell'altruismo. A causa dell'associazione con il fuoco, in alcune culture lo collegano alla violenza o ai demoni e all'inferno. Essendo un colore che salta subito all'occhio, si usa spesso sui veicoli dei pompieri, mentre nel traffico è segno di pericolo e invita alla cautela. L'associazione a sentimenti e valori positivi (potenza e orgoglio) è testimoniata dalla sua presenza in molte bandiere nazionali (Olesen 2019). In Slovenia il rosso è considerato un colore femminile: i maschi vestiti di rosso attirano l'attenzione.

\section{LE ESPRESSIONI IDIOMATICHE}

Il sistema lessicale di ogni lingua è costituito oltre che da parole singole anche da sequenze e combinazioni di parole sufficientemente stabili che vengono recuperate dalla memoria $\mathrm{e}$ impiegate come unità semantiche. Si tratta di forme lessicali complesse che costituiscono il sistema fraseologico di una lingua. All'interno dei fenomeni fraseologici occupano un posto rilevante le espressioni idiomatiche o modi di dire; si tratta di brevi sequenze fisse di parole dotate di un significato specifico, figurato e non riconducibile alla somma dei significati delle parole che li compongono. Espressioni come essere al verde, andare in bianco, mettere nero su bianco, vedere nero, vedere rosso non significherebbero nulla se considerate solo come somma dei significati dei loro componenti; considerate, invece, globalmente rimandano a un significato traslato, risultato di procedimenti metaforici e condiviso dall'intera comunità linguistica.

I modi di dire sono, per lo più, metafore o immagini tratte dalle esperienze di vita. La metafora è lo strumento principe idoneo a traslare gli oggetti dal concreto al mondo psichico, dove assumono un nuovo referente comprensibile più o meno rapidamente dai parlanti. Alcune immagini metaforiche sono così presenti nell'uso quotidiano del parlare da perdere la vivacità originaria ed essere percepite come normali parole complesse e non come espressioni della creatività umana.

Esiste una relazione fra significato letterale e significato idiomatico, e dunque è possibile spiegare perché un modo di dire abbia un certo significato idiomatico, riconducendolo a una struttura metaforica generale che dà senso all'associazione tra quell'espressione e il suo significato convenzionale. Questa regolarità, tuttavia, non significa che tutti i modi di dire siano descrivibili attraverso metafore generali; una spiegazione locale è talvolta l'unica possibile per alcuni modi di dire di origine storica, letteraria, geografica, mitologica e quant'altro. 
Anche se ancora non si è giunti ad una descrizione sistematica e teorica dei modi di dire, né ad una loro classificazione omogenea, gran parte degli studiosi concorda nell'affermare che le proprietà che definiscono un'espressione come idiomatica sono la fissità, la non composizionalità e l'opacità semantica.

La fissità, o grado di congelamento sintattico, è la caratteristica attribuita alle espressioni idiomatiche dal punto di vista lessicale e sintattico, che le oppone alle frasi libere. Infatti, nelle locuzioni idiomatiche si può notare una certa stabilità semantico-sintattica in quanto non sempre è accettabile sostituire i componenti lessicali con altri, anche se sinonimici. La fissità appare la caratteristica che maggiormente distingue le espressioni idiomatiche da quelle libere.

Le espressioni idiomatiche sono caratterizzate anche da vari gradi di opacità semantica, che ne determina il livello di idiomaticità e le differenzia dalle espressioni letterali, semanticamente trasparenti. Trasparenza e opacità semantica definiscono il grado di facilità o di difficoltà con cui il significato idiomatico può essere ricavato grazie all'immagine evocata. Nel caso in cui l'espressione non evochi alcuna immagine che permetta di risalire al significato figurato, essa viene definita opaca. È interessante notare che le espressioni con il maggior grado di opacità sono anche le meno modificabili. Le espressioni opache hanno spesso alla base una motivazione di tipo storico o culturale e che in molti casi è andata perduta, e non può essere direttamente percepita dai parlanti.

Tendenzialmente, tuttavia, si può dire che le espressioni idiomatiche presentano un basso grado di trasparenza, in quanto l'interpretazione non è direttamente deducibile o addirittura non è possibile senza l'ausilio di conoscenze esterne o senza la presenza di un preciso contesto sia linguistico che extralinguistico.

\section{METODOLOGIA}

Il lavoro si è articolato in due fasi distinte, la prima è consistita nella raccolta del materiale e si è basata sulla ricerca nei dizionari e siti dedicati alle espressioni idiomatiche in lingua slovena, italiana e greca. Per lo sloveno è stato consultato il Dizionario delle espressioni idiomatiche (Keber 2011) e un sito $^{2}$; per l'italiano si è consultato vari dizionari delle espressioni

${ }^{2} \mathrm{http}: / / w w w . f r a z e m i . c o m / f r a z e m \_s e z n a m . p h p ? f r a z e m \_s t r a n=2$ (consultato il 10 settembre 2019) - Questo è un portale interattivo in cui c'è un vocabolario dove i linguisti includono tutte le espressioni idiomatiche con relativa spiegazione del significato, etimologia, esempi concreti di uso e traduzioni in più lingue europee. È stato consultato perché, data la natura molto vivace nel creare neologismi in questo campo, molte espressioni 
idiomatiche (Lurati 2002; Pittano 1992; Quartu 1993 e Sorge 2001) e per

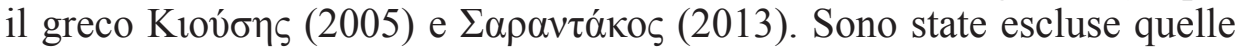
espressioni idiomatiche classificate come arcaiche e non più in uso nella lingua viva, mentre sono rimaste quelle appartenenti a particolari ambiti professionali (ad es. linguaggio giornalistico, della finanza ecc.). Quando un'espressione idiomatica contiene due o più cromonimi, viene classificata nel gruppo del primo cromonimo che appare nella sequenza sintagmatica dell'espressione. Dopo aver costruito un corpus trilingue di tutte le espressioni idiomatiche con bianco, nero e rosso, esse sono state annoverate in uno dei 5 gruppi (I-V) in base alle somiglianze tra le lingue:

I. Espressioni uguali in tutte e tre le lingue;

II. Espressioni uguali in italiano e sloveno;

III. Espressioni uguali in italiano e greco;

IV. Espressioni uguali in greco e sloveno;

V. Espressioni presenti in una sola lingua.

Dopo aver raccolto e classificato le espressioni idiomatiche, queste sono state tramutate in dati numerici a loro volta trasformati in percentuali con l'intento di scoprire il grado di convergenza tra le singole lingue. Questo quadro è stato comparato con i dati etimologici per poter provare il grado di influenza dello sviluppo diacronico sulle effettive similitudini sincroniche.

La seconda parte ha avuto come obiettivo un'analisi qualitativa del corpus in cui è stato prima fatto un elenco delle accezioni diverse che ogni singolo cromonimo può avere, per poi verificare come si lega ai valori simbolici e se le lingue le condividono. Infine è stata fatta un'analisi contrastiva con commento su alcune peculiarità legate ai falsi amici e alla polisemia.

\section{ANALISI QUANTITATIVA}

In tutto sono state registrate 157 espressioni idiomatiche diverse, di cui 38 , ovvero il $24 \%$ sono uguali in tutte e tre le lingue. La maggiore similarità si nota tra lo sloveno e il greco, che hanno 15 espressioni idiomatiche in comune (10\%). L'italiano e il greco ne hanno $13(8 \%)$ e lo sloveno e l'italiano sono i meno simili con il 4\% (solamente 7 espressioni idiomatiche). Il 54\% delle espressioni, invece, figura in una sola lingua.

idiomatiche non sono presenti nel dizionario di Keber del 2011, ma le possiamo trovare in questo sito. 


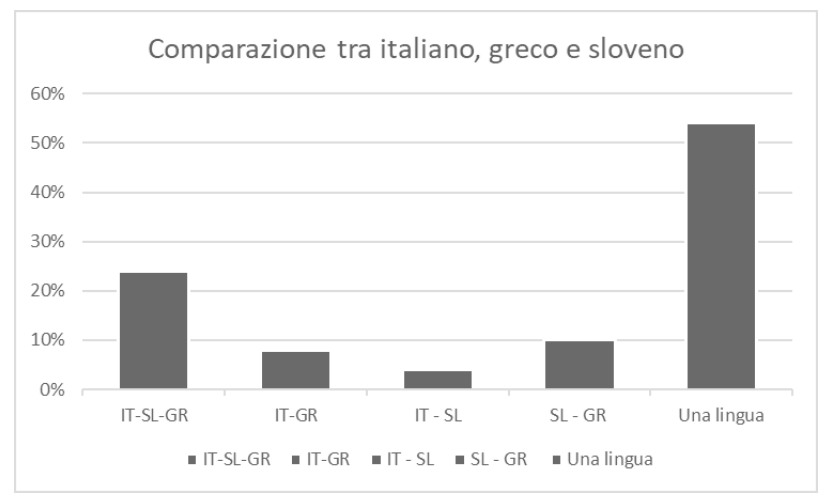

Grafico 1. Convergenze e divergenze statistiche tra italiano, sloveno e greco

Dall'analisi statistica possiamo desumere che più della metà delle espressioni idiomatiche contenenti i cromonimi nero, bianco e rosso divergono, mentre solamente un quarto di esse converge. Nel dettaglio vediamo che combacia il 24\% delle espressioni idiomatiche contenente nero, il 18\% di quelle contenenti bianco e il $36 \%$ di quelle con rosso. Tale quadro (una percentuale leggermente più alta per quanto riguarda il rosso) potrebbe confermare l'ipotesi che l'etimologia comune di rosso, derivata dalla stessa radice indoeuropea in tutte e tre le lingue, influisce su una maggiore somiglianza. Pur essendo la simbologia molto simile, le percentuali delle espressioni legate ad una sola lingua mostrano che le tre lingue, pur derivando da una base comune, hanno preso direzioni diverse nella creazione di espressioni idiomatiche.

\section{ANALISI QUALITATIVA E DISCUSSIONE}

La simbologia influisce sulla creazione di espressioni idiomatiche, quindi i valori simbolici dei cromonimi si riflettono nel significato figurato che gli attribuiscono i locutori di una determinata cultura e, di conseguenza, anche di quella lingua. La comparazione delle accezioni emerse dall'analisi con quelle nei dizionari delle lingue in questione mostra più o meno la stessa sfaccettatura.

\subsection{Nero}

In merito al cromonimo nero possiamo sostenere che nell'uso troviamo soprattutto i valori simbolici negativi ('morte nera', 'lavoro in nero'), un solo caso di valore legato a una corrente politica conservativa ('camicie 
nere' come sinonimo di fascisti in sloveno e italiano) $)^{3}$ e alcuni casi di valore neutrale ('oro nero'). I valori positivi, di nascita più recente, che indicano eleganza, non si riflettono (ancora) nelle espressioni idiomatiche. Le più recenti ad essere entrate nella lingua sono dei calchi dall'inglese, come ad esempio il black Friday che in italiano preserva la forma originale, dato che 'venerdì nero' si associa a una cosa negativa. In sloveno e greco viene

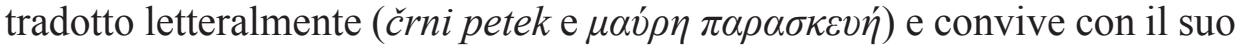
omonimo che ha le radici nel passato più remoto, quando ancora si accostava a un giorno che veniva considerato particolarmente negativo (slo. črni četrtek, črni petek).

Benché il cromonimo bianco sia quello più frequentemente usato in sloveno e greco, dall'analisi del corpus si evince che il nero appare, numericamente, in più espressioni idiomatiche. Conclusione questa a cui giungono anche Schmiedtová e Schmiedtová (2000) in uno studio svolto sulla lingua ceca in cui provano che il nero è semanticamente più complesso rispetto al bianco. A questo proposito avanziamo l'ipotesi che il nero, negativo, sia psicologicamente più attraente rispetto al bianco, considerato positivo.

Quando 'nero' si colloca con nomi di per sé negativi ne amplia la negatività ('nero come la fame/l'anima di Giuda/il di dentro di un infedele'), mentre accostato a nomi neutri li rende negativi ('giornata nera').

Proponiamo di seguito le accezioni comuni a tutte e tre le lingue che sono state riscontrate:

I. Assenza di colore, l'opposto di bianco (n. su bianco, n. come il carbone, scatola n.)

II. Un colore scuro, ma non necessariamente nero (occhio n., nuvole n., slo. risati v črnih barvah 'dipingere con colori neri' - per descrivere qualcuno o qualcosa in maniera estremamente negativa)

III. Di popolazione che ha la pelle scura (Continente n.)

IV. Crudele (humor n.)

V. Sudicio, sporco (n. come uno spazzacamino, slo. kolikor je za nohtom črnega 'quanto c'è di n. dietro a un'unghia' - per dire niente o molto poco in senso negativo)

VI. Doloroso, pieno di angoscia e afflizione (giornata n., morte n.)

3 'Camicie nere' è una metonimia associata a chi in passato aderì alla corrente politica del fascismo che nasce come una legittima corrente politica conservatrice con connotazioni puramente neutrali. Oggi il termine nella cultura slovena evoca un periodo storico negativo e quindi il calco dall'italiano črne srajce e la conseguente derivazione črnosrajčniki nel Dizionario delle espressioni idiomatiche (Keber 2011) viene descritta con la marca d'uso 'peggiorativo'. 
VII. Di persona con la coscienza macchiata di gravi colpe o di comportamento moralmente riprovevole, vergogna (anima n., slo. črni

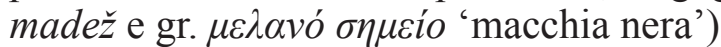

VIII. Qualcosa di negativo, legato alla morte e ai delitti (lista n., libro n., pecora n., cronaca n., slo. črna točka 'punto n.' riferito a un tratto di strada particolarmente pericoloso in cui sono successi molti incidenti gravi)

IX. Depressione, stato d'animo pessimistico (vedere tutto n.)

X. Qualcosa di illegale e/o disonesto (mercato/borsa n., fondi n., lavoro in n.)

XI. Malvagio, cattivo, brutto (magia n., slo. hudič ni tako črn, kot ... 'il diavolo non è così nero come...' - considerare qualcosa in maniera più negativa di quanto lo sia realmente)

XII. Molto grande in senso negativo (n. ingratitudine)

La maggioranza delle accezioni combacia nelle tre lingue, mentre divergono due, la XIII che si usa solamente in italiano e sloveno (data la condivisione territoriale di questo periodo storico), e la XIV che richiama all'arrabbiatura, ma solamente in italiano e greco.

XIII. Simbolo del fascismo (camicie $\mathrm{n}$.)

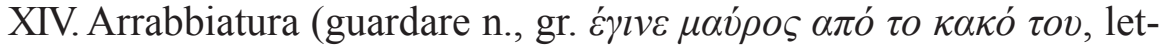
teralmente 'diventare $\mathrm{n}$. dalla propria malizia')

Vanno inoltre aggiunti a quest'elenco alcuni casi isolati in cui il cromonimo assume un significato particolare, come ad esempio il gr. $\mu \alpha v ́ \rho \alpha$ $\mu \alpha ́ \tau \iota \alpha$ 'occhi neri' che viene usato quando non si vede una persona da molto tempo per cui gli occhi appaiono scurissimi dalla lontananza o almeno così sembrano. In sloveno esiste l'espressione zadeti v črno 'colpire nel nero' che nasce dal tiro al segno e significa 'fare la mossa giusta o trovare il nocciolo della questione'.

\subsection{Bianco}

Se da una parte il nero ha prevalentemente significato negativo (è positivo ad esempio nell'espressione slovena črne številke 'numeri neri' che in opposizione a rdeče številke 'numeri rossi' indica un bilancio positivo) e tende a trasferirlo sul nome cui fa riferimento, il bianco, dall'altra parte, tende a rendere positivo il nome cui si accosta, anche se questo è inerentemente negativo (si pensi ad es. all'espressione 'bugia bianca'), ma anche in generale (es. 'pecora nera' e 'mosca bianca'4). Un'eccezione a questa tendenza è l'espressione in uso nella lingua giornalistica in sloveno beli slon

${ }^{4}$ Sl. bela vrana ‘corvo bianco' riferito a persona rara, quasi unica per le sue qualità. 
'elefante bianco' che si riferisce a una grande impresa con enormi perdite finanziarie. Questa espressione è un calco dall'inglese white elephant che significa un regalo o cosa il cui mantenimento è più caro del suo valore $\mathrm{e}$ deriva dalle culture asiatiche (Keber 2011) ${ }^{5}$, dove il bianco ha una simbologia negativa. Quando si accosta a 'morte' ('morte bianca'), non riesce a migliorarne il significato, ma ne specifica soltanto la causa. Se per esempio la compariamo con 'morte nera' (la peste) possiamo desumere che si tratta di due lessicalizzazioni diverse.

I significati più frequenti assunti da 'bianco' che accomunano tutte e tre le lingue sono:

I. il colore bianco (b. come la neve/il latte/il giglio)

II. qualcosa di chiaro ma non necessariamente bianco (b. come un morto/come la tela, slo. come la cera/la parete/la rapa, pane b.)

III. collegato alla neve, al Natale e più in generale all'inverno e al freddo (continente b., b. Natale, settimana b., slo. circo b.)

IV. pallido (b. come uno straccio)

V. nocivo; collegato alla droga o altra sostanza di questo colore (polvere b.)

VI. buono, positivo e anche raro (bugia b., mosca b., merlo b.)

VII. resa (bandiera b.)

VIII. rifiuto di collaborazione (sciopero b.)

Oltre alle accezioni comuni c'è una solo slovena (IX), una solo italiana e greca $(\mathrm{X})$ e tre slovene e greche (XI-XIII):

IX. la luce mediana del giorno (pri belem dnevu 'al b. giorno', jasno kot beli dan 'chiaro come il b. giorno', ugledati beli dan 'intravedere il b. giorno - nascere')

$X$. assenza di qualcosa, fiasco (notte b., andare in b., cambiale in b., carta b., mangiare in b., voto b.)

XI. resistenza (avere il fegato b.)

XII. razza umana (merce b., carne b.)

XIII.rabbia (guardare con il b. dell'occhio)

Come nel caso di 'nero', anche con 'bianco' la maggioranza delle accezioni è uguale in tutte e tre le lingue. In italiano ha preservato il significato primario, cioè 'luccicante' nell'espressione 'armi bianche' (Lurati 2002), ma i parlanti oggi non percepiscono più quest'accezione. Il significato che vede bianco collegato alla neve e a ciò che riguarda gli sport sulla neve ha più espressioni in sloveno, perché lo sci è lo sport nazionale della Slo-

${ }^{5} \mathrm{Nel} \mathrm{Siam}$, in Laos e in Kambodia un elefante bianco è un animale sacro che porta pioggia. Il re del Siam che intendeva distruggere qualche suo suddito gli regalava un elefante bianco sacro il cui mantenimento fu talmente caro che lo portava alla rovina. 
venia. Di conseguenza numerose espressioni tendono a nascere in questo dominio semantico che funge da centro di espansione e attrazione a livello nazionale (Sperber 1965). Sia 'circo bianco' che 'carovana bianca' sono espressioni tratte dal gergo dei commentatori sportivi e diffusesi nella lingua standard.

In più espressioni idiomatiche presenti in italiano e in greco '(in) bianco' significa assenza o mancanza di qualcosa ('carta bianca', 'cambiale in bianco', 'notte in bianco', 'matrimonio bianco' ecc.). Nella maggioranza dei casi implica una sfumatura negativa, ma può avere anche un significato neutro come ad esempio in 'mangiare in bianco'. È interessante l'espressione in sloveno e greco moderno tradotta alla lettera 'guardare con il bianco' che equivale all'italiano 'avere gli occhi fuori dalle orbite' e significa letteralmente avere un'espressione talmente distorta dall'ira da far pensare a una fuoriuscita degli occhi dalla loro sede (cfr. 'guardare rosso'). Questa mimica facciale è conosciuta in tutte e tre le culture, ma cambia la prospettiva con cui viene descritta.

L'espressione italiana 'far venire i capelli bianchi' nello sloveno esiste, ma si usa con il cromonimo 'grigio' (delati sive lase 'fare venire i capelli grigi'). Tuttavia, anche lo sloveno si serve di un verbo deaggettivale per esprimere la stessa cosa: beliti si glavo (trad. let. 'imbiancarsi la testa') che significa pensare tanto ai problemi e quindi preoccuparsi eccessivamente fino a farsi venire i capelli bianchi. Anche questo è un esempio di come per lo stesso fenomeno cambia prospettiva.

Il bianco appare anche nell'espressione slovena e greca princ na belem

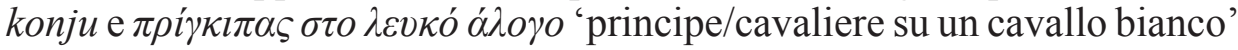
che in italiano si traduce con 'principe azzurro'. La causa di tale divergenza risiede nell'etimologia delle espressioni. Quella comune allo sloveno e al greco deriva da una base fiabesca in cui un principe su un cavallo bianco salva la principessa da una situazione di disagio, mentre in italiano gli studiosi non sono ancora unanimi sull'etimologia di questa locuzione che deriverebbe dal francese prince Azur, dove fu registrata già nel $1854 \mathrm{e} \mathrm{da}$ qui sarebbe passata all'italiano (prima attestazione nel 1898) e allo spagnolo (D’Achille 2011).

Come vediamo, la struttura semantica di bianco è meno complessa di nero nelle tre lingue analizzate. Infatti mancano le accezioni opposte al nero inteso come 'illegale' e 'pessimistico'; spesso le espressioni come 'notizia nera' (negativa) non trovano una controparte in 'notizia bianca' (positiva). Quando ha significato di neve ('Natale bianco') in sloveno si contrappone a verde (sl. zelena zima 'Natale verde' cioè senza neve). Inoltre il bianco assume tutta una serie di connotazioni negative ('resa', 'nocivo', 'fiasco' e 'mancanza'), cosa che non viene descritta dalla simbologia e quindi ha origini diverse. 


\subsection{Rosso}

Il rosso è fedele alla simbologia descritta nel paragrafo 2.2 e appare nelle espressioni idiomatiche con le seguenti accezioni:

I. il colore, spesso delle labbra (r. come il fuoco/la brace/il sangue/un gambero/un pomodoro/peperone/rubino/papavero...; croce rossa, sl. filo rosso, sl. gallo rosso)

II. di persona, colorito della pelle del volto per indicare vergogna, imbarazzo, timidezza o troppa esposizione al sole

III. pericolo (allarme r.)

IV. trasgressione, sesso (luci r., telefono r.)

V. prestigio (tappeto $r$.)

VI. debito (numeri $r$, andare in $\mathrm{r}$.)

VII. ira (r. dall'ira, vedere r.)

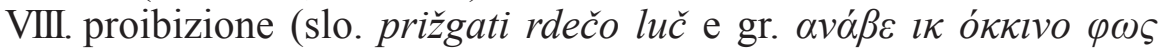
'accendere la luce r.', it. dare il disco r.)

IX. punizione (dare il cartellino r.)

Le accezioni elencate per il rosso combaciano sia con la simbologia sia con le definizioni nel dizionario e sono quasi uguali in tutte e tre le lingue. Le differenze si manifestano a livello lessicale, ad esempio se 'rosso come il fuoco/sangue/gambero/papavero' figura in tutte, l'espressione 'rosso come un tacchino' è solo slovena, 'rosso come una ciliegia/il sedere di una scimmia' solo greca e 'rosso come un peperone/rubino/una fragola' solo italiana. In accordo con la simbologia, i significati sono sia positivi che negativi, anche se prevalgono quelli negativi (proibizione, trasgressione, ira, debito, punizione). Questa tendenza al negativo è stata avvertita anche nell'analisi di bianco come simbolo del bene, mentre il nero non ha subito simili cambiamenti in direzione della simbologia positiva.

Notiamo in tutte e tre le lingue l'espressione 'tappeto rosso', importata dall'inglese, che in italiano spesso viene usata anche nella forma originale red carpet, mentre le altre due lingue si servono di calchi sintattici.

I significati X e XI esistono solo in sloveno, mentre XII solamente in italiano:

X. incendio (nell'espressione rdeči petelin 'gallo rosso') ${ }^{6}$

${ }^{6}$ L'espressione slovena originerebbe dal fatto che molte genti, tra cui anche gli slavi, in passato ritenevano il gallo simbolo del fuoco e del sole. Per questo motivo lo sacrificavano al proprio dio. Una delle etimologie più antiche di questa espressione sostiene che il gallo, a causa del suo colore della brace e dell'occhio scintillante, fosse ritenuto il simbolo delle vampe (Snoj 2016). 
XI. l'essenza di qualcosa (rdeča nit 'il filo rosso"7 $)$ che in italiano viene usato come 'filo conduttore'.

XII. consulenza medica relativa alla gravidanza (telefono $\mathrm{r}$.)

Il rosso simboleggia anche un movimento politico (dei lavoratori, del comunismo e del socialismo), ma questo non si riflette nelle espressioni idiomatiche. Tuttavia le lingue lo inseriscono nel lessico in modi diversi (es. in italiano nel titolo dell'inno ai lavoratori 'Bandiera rossa' e in sloveno in rdeči 'i rossi' con riferimento ai socialisti, comunisti e in generale politici della sinistra in opposizione a beli 'i bianchi' come rappresentanti dei controrivoluzionari o i politici della destra).

\subsection{Polisemia e falsi amici}

Alcuni fenomeni interessanti sono i casi di polisemia di un'espressione idiomatica. Ad esempio lo slo. gledati črno 'guardare nero' ha due accezioni distinte: la prima significa essere arrabbiato e la seconda essere pessimista. Similmente l'italiana 'peste bianca' può significare morte causata dalla tubercolosi e natalità negativa nei paesi europei. In chiave interlinguistica i casi più interessanti sono quelli in cui la stessa espressione in tutte e tre le lingue si copre semanticamente solo parzialmente come nel caso di 'morte

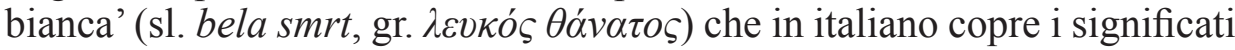
$\mathrm{I}-\mathrm{IV}$, in sloveno il III e il $\mathrm{V}$ e in greco solamente il V.

I. sindrome della morte improvvisa del lattante;

II. incidente mortale che si verifica sul posto di lavoro;

III. decesso ad alta quota a causa del freddo o della neve (slavine);

IV. soprannome del cecchino finlandese Simo Häyhä e

V. polvere di colore bianco che nuoce alla salute.

L'ultimo esempio ci porta ai falsi amici tra le espressioni idiomatiche. Si pensi a 'camicie nere' (sl. črne srajce) che in italiano e sloveno designa gli appartenenti al regime fascista. In greco invece, la corrispondente espressione fa riferimento agli abitanti dell'isola di Creta, dove la camicia nera fu introdotta come capo d'abbigliamento quotidiano nel 1936. Anche 'treno bianco' (adibito al trasporto di ammalati in pellegrinaggio) e il corrispettivo sloveno beli vlak (treno adibito al trasporto degli sciatori sulle piste da sci) sono dei falsi amici in quanto hanno la stessa forma in traduzione, ma significati totalmente diversi. Un altro esempio è l'espressione 'telefono rosso' presente in tutte e tre le lingue con valori diversi. In greco si riferisce a una linea erotica e quindi si classifica nell'accezione IV (trasgressione), in

${ }^{7}$ Quest'espressione sarebbe entrata nella lingua slovena solamente dopo la Seconda guerra mondiale come calco dal tedesco der rote Faden (Snoj 2016). 
italiano si usa con valore neutrale per indicare una linea telefonica dedicata a future e neo mamme (XII - consulenza medica legata alla gravidanza) e in sloveno alla linea tra Washington e Mosca ai tempi della guerra fredda (in italiano linea rossa) ricollegabile all'accezione III (pericolo, emergenza).

\section{CONCLUSIONI}

Dal punto di vista fisico tutti gli esseri umani percepiscono i colori allo stesso modo, quindi il fatto di avere un diverso numero di espressioni idiomatiche contenenti cromonimi in lingue diverse rientra nell'ambito cognitivo e non visivo. Un confronto interlinguistico di espressioni idiomatiche contenenti bianco, nero e rosso tra l'italiano, lo sloveno e il greco porta alla conclusione che esistono molte più divergenze che convergenze. In primo luogo, l'analisi etimologica dei termini fa emergere che solamente il rosso si può ricondurre alla stessa radice indoeuropea, mentre il bianco e il nero hanno origini da radici indoeuropee diverse. L'analisi quantitativa porta alla conclusione che più della metà delle espressioni idiomatiche appare solamente in una lingua, un quarto in tutte e tre e che c'è più convergenza tra lo sloveno e il greco, meno tra l'italiano e il greco e minore ancora è la corrispondenza tra lo sloveno e l'italiano. Di conseguenza la contiguità territoriale, culturale e religiosa non influisce su una maggiore equivalenza. La simbologia si riflette nelle espressioni idiomatiche anche se non tutti i valori simbolici vi trovano posto.

Ci sono vari esempi di polarizzazione del significato di nero come negativo e bianco come positivo. I casi in cui nero assume un significato neutro sono pochi, mentre il bianco e il rosso hanno più accezioni negative. Si nota anche l'influenza dell'inglese, però in questi casi l'italiano mostra una predilezione ai prestiti non adattati, mentre lo sloveno e il greco ricorrono ai calchi di traduzione. Ci sono, inoltre, alcuni interessanti casi di polisemia e falsi amici che riflettono la vivace e creativa natura delle espressioni idiomatiche che interlinguisticamente mostrano un quadro sfaccettato e complesso. Dall'analisi è emerso, inoltre, che la frequenza d'uso non pregiudica necessariamente il numero di espressioni idiomatiche.

\section{BIBLIOGRAFIA}

Banfi, E. e Grandi, N. (2003). Lingue d'Europa. Elementi di storia e di tipologia linguistica. Roma: Carocci.

Basile, G., Casadei, F., Lorenzetti, L., Schirru, G. e Thornton, A. M. (2010). Linguistica generale. Roma: Carocci. 
Berlin, B., e Kay, P. (1969). Basic Color Terms: Their Universality and Evolution. Berkeley \& Los Angeles: University of California Press.

CoLFIS http://esploracolfis.sns.it/EsploraCoLFIS/\#! $0: \mathrm{f}=1 ; 1: \mathrm{fr}=$ nero\&r=T (12. 9. 2019)

CORIS http://corpora.dslo.unibo.it/coris_ita.html

http://www.frazemi.com/frazem_seznam.php?frazem_stran=2 (10. 9. 2019)

Corpus La Repubblica https://corpora.dipintra.it/public/run.cgi/first?corpna $\mathrm{me}=$ repubblica\&reload $=1 \&$ iquery $=\&$ queryselector $=$ lemmarow $\&$ lemm $\mathrm{a}=\&$ phrase $=\&$ word $=\& \mathrm{char}=\& \mathrm{cql}=\&$ default attr $=$ word $\& \mathrm{fc}$ lemword window type $=$ both $\&$ fc_lemword_wsize $=5 \& \mathrm{fc}$ lemword $=\& \mathrm{fc}$ 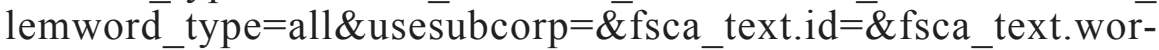 dcount $=(15.9 .2019)$

D’Achille, P. (2011). Prosoponimi fiabeschi: Cenerentola, Biancaneve, la Bella Addormentata e il Principe Azzurro. In E. Caffarelli e M. Fanfani (a cura di), Lo spettacolo delle parole Studi di storia linguistica e di onomastica in ricordo di Sergio Raffaelli (pp. 501-523). Supplemento al $n^{\circ}$ XVII, 1 - primo semestre 2011, della Rivista Italiana di Onomastica. Roma: Società Editrice Romana.

Daniele, V. (2015). EtimoItaliano: il significato originale di parole e parolone... nella lingua italiana. Testo disponibile al sito: https://www. etimoitaliano.it/ (4. 9. 2019)

Gigafida. Korpus slovenskega jezika (2007): http://www.gigafida.net (15. 9. 2019)

Kay, P. e Maffi, L. (2013). Number of Basic Colour Categories. In Dryer, M. S. e Haspelmath, M. (a cura di), The World Atlas of Language Structures Online. Leipzig: Max Planck Institute for Evolutionary Anthropology. Testo disponibile al sito: https://wals.info/chapter/133 (10. 9. 2019).

Keber, J. (2011). Slovar slovenskih frazemov. Ljubljana: Založba ZRC, ZRC SAZU.

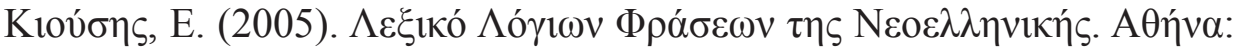
'Evvoia.

Kocjančič, S. (2019). Slovenski, italijanski in angleški frazemi s področja barv. Tesi di laurea magistrale. Koper: Unierza na Primorskem.

Kovačev, A. N. (1997). Govorica barv. Ljubljana: Prešernova družba.

Lurati, O. (2002). Per modo di dire: storia della lingua e antropologia nelle locuzioni italiane ed europee. Bologna: CLUEB.

Mattevi, A. (2013). L'etimologia dei colori. Lineagrafica Bertelli Editori S.n.c. Testo disponibile al sito: http://www.lineagraficabertelli.it/letimologia-dei-colori/ (10. 9. 2019). 


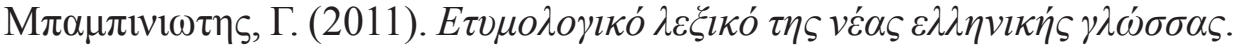

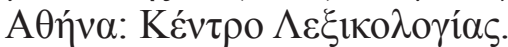

Musek, J. (1990). Simboli, kultura, ljudje. Ljubljana: Znanstveni inštitut Filozofske fakultete.

Olesen, J. (2019). Red Color Meaning. Testo disponibile al sito: https://www. color-meanings.com/red-color-meaning-the-color-red/ (10.9.2019).

Nocentini, A. (2004). L'Europa linguistica. Profilo storico e tipologico. Firenze: Le Monnier.

Philip, S. G. (2003). Collocation and connotation: A Corpus-based Investigation of Colour Words in English and Italian. PhD Thesis. Birmingham: University of Birmingham.

Pittano, G. (1992). Frase fatta capo ha. Dizionario dei modi di dire, proverbi e locuzioni. Bologna: Zanichelli.

Quartu, B. M. (1993). Dizionario dei modi di dire della lingua italiana: 10.000 modi di dire ed estensioni figurate in ordine alfabetico per lemmi portanti e campi di significato. Milano: Rizzoli.

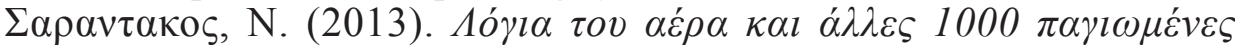

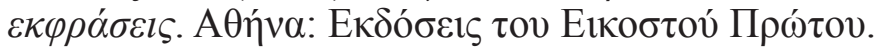

Schmiedtová, V. e Schmiedtová, B. (2000). The color spectrum in language: The case of Czech: Cognitive concepts, new idioms and lexical meanings. Proceedings of The 10th International Symposium on Lexicography, 285-292. Testo disponibile al sito: https://www.researchgate. net/publication/27269304 The color spectrum in language The case_of_Czech_Cognitive_concepts_new_idioms_and_lexical_meanings $(\overline{6} .11 .2019)$

Snoj, M. (2016). Slovenski etimološki slovar. Ljubljana: Založba ZRC.

Sorge, P. (2001). Dizionario dei modi di dire della lingua italiana: origine e significato delle frasi idiomatiche e delle forme proverbiali rare e comuni. Roma: Newton \& Compton editori.

Sperber, H. (1965). Einführung in die Bedeutungslehre. Bonn: Dümmlers Ferlag.

Zeroníková, M. (2009). Barve v češki in slovenski frazeologiji. (Bakalarsko diplomsko delo). Brno: Masarykovauniverzita, Filozofickáfakulta. Testo disponibile al sito: https://is.muni.cz/th/ix3t3/Zeronikova_THESIS. pdf (28.8.2019) 


\section{SOME CHROMONYMS IN IDIOMATIC EXPRESSIONS IN ITALIAN, GREEK AND SLOVENIAN: CONVERGENCES AND DIVERGENCES}

\section{Summary}

Although Italian, Slovenian and Greek belong to different language groups, they all have the same common ancestor, Indo-European. The article presents the etymological origins, symbolism, influence and cultural contiguity manifested in idiomatic expressions with chromonyms from a contrastive analysis perspective. The objective is to investigate which of these criteria could have had more influence on meaning and which languages have the greatest similarities. This research takes into account the three most frequently used chromonyms based on linguistic corpora, namely black, white and red and their translations in all three languages. The analysis leads to the conclusion that territorial, cultural and religious contiguity did not matter much, nor did etymology. The three languages share basic symbolism, but in most cases, they differ in their use. Slovenian and Greek, the most distant languages territorially and culturally, which have no direct contact, find the greatest number of similarities. This is followed by the pair of Italian and Greek languages and finally Slovenian and Italian, which have the fewest similarities.

Keywords: contrastive phraseology, chromonyms, idiomatic expressions, Slovenian, Greek, Italian. 